1Departamento de Promoción de la Salud de la Mujer y el Recién Nacido, Escuela de Obstetricia. Facultad de Medicina, Universidad de Chile; Santiago, Chile.

2Departamento de Salud Pública, Facultad de Medicina, Pontificia Universidad Católica de Chile; Santiago, Chile.

${ }^{3}$ Departamento de Epidemiología Nutricional, Instituto de Nutrición y Tecnología de los Alimentos (INTA), Universidad de Chile; Santiago, Chile. ${ }^{4}$ Facultad de Medicina, Universidad de Chile, Santiago, Chile.

${ }^{5}$ Departamento de Pediatría, Facultad de Medicina, Pontificia Universidad Católica de Chile. aEnfermera-Matrona. bLicenciada. 'Estadístico.

Recibido el 4 de septiembre de 2014, aceptado el 22 de octubre de 2014.

Correspondencia a: Marcela Araya Bannout Dirección postal: Avenida Independencia 1027, Independencia.

Código Postal 8389100 clasificador 7, Santiago, Chile.

Teléfonos: 56229876856 marcelaaraya@med.uchile.cl

\section{Obesidad en mujeres chilenas en edad fértil}

\author{
MARCELA ARAYA B. MPH, PhD ${ }^{1, a, b}$, OSLANDO PADILLA ${ }^{2, c}$, \\ MARÍA LUISA GARMENDIA MPH PhD ${ }^{3}$, \\ EDUARDO ATALAH MPH ${ }^{4}$, RICARDO UAUY PhD ${ }^{3,5}$
}

\section{Prevalence of obesity among Chilean women in childbearing ages}

Background: Women in childbearing ages (WCBA) represent a strategic intervention group to reverse the global trend of increasing obesity. Aim: To conduct a review of studies reporting obesity prevalence in WCBA in Chile in the last 25 years. Material and Methods: To describe obesity prevalence, we used three approaches, namely qualitative review of studies describing obesity prevalence in WCBA and a comparative study of the prevalence of obesity, overweight, abdominal and morbid obesity in WCBA from 2003 and 2009-10 National Health Surveys (ENS). Finally, nutrition status trends of pregnant women in the period 1987-2013 were plotted. Results: According to ENS, obesity prevalence exceeded 20\% and excess weight 50\%, both in 2003 and 2009-10 surveys. We found seven population studies, being difficult to compare them due to the heterogeneity of obesity definitions, design, and populations. Population studies showed that the prevalence of obesity increases along with age. The higher prevalence of obesity in WCBA aged 20 to 43 years was found in the city of San Carlos in 2007 (35.5\%). The lowest was found in female university students, ranging from $0 \%$ to $5.9 \%$. Obesity in pregnant women increased from $12.9 \%$ to $32.2 \%$ in the period 1987-2004 and from $20.3 \%$ to $26.3 \%$ in the period 2005 2013. Conclusions: There is a high and increasing prevalence of obesity among women in childbearing age, whether pregnant or not-pregnant.

(Rev Med Chile 2014; 142: 1440-1448)

Key words: Chile; Obesity; Pregnancy; Women.
L a obesidad en la mujer en edad fértil (MEF) es de particular importancia por sus consecuencias a la salud tanto en el mediano como en largo plazo. Durante el embarazo, la obesidad aumenta la probabilidad de desarrollar diabetes gestacional, hipertensión arterial y preeclampsia $^{1-3}$ y de tener un parto por cesárea ${ }^{1,4-7}$, entre otras complicaciones. En el largo plazo, la obesidad se asocia a problemas reproductivos ${ }^{8} y$ al desarrollo de enfermedades crónicas no transmisibles (ECNT), tales como hipertensión arterial, diabetes tipo II, dislipidemias ${ }^{9}$ y algunos tipos de cáncer ${ }^{10,11}$. Pero las consecuencias de la obesidad se extienden más allá de la mujer, a su descendencia. Los recién nacidos de madres obesas durante el embarazo presentan más complicaciones en el período perinatal (nacer grande para la edad gestacional (GEG), macrosómico, pre-término ${ }^{1,4,6,12-14}$, etc.). Al mismo tiempo, un ambiente obesogénico durante los períodos pre-concepcional y gestacional, incrementará en el niño el riesgo de desarrollar en el largo plazo obesidad y ECNT del adulto ${ }^{15-16}$.

La prevalencia de obesidad en las MEF de 2049 años aumentó en el mundo a partir de 1980. Destacan las regiones de las Américas y el Caribe y Oceanía por presentar las prevalencias más altas 
de obesidad; mientras ésta fue inferior a $10 \%$ en la década 1980-89, ambas regiones presentaron prevalencias de obesidad superiores a $20 \%$ el año $2008^{17}$. La serie de estudios NHANES realizados en Estados Unidos de Norteamérica desde 1960 en adelante, demostraron que en mujeres de 20-39 años, la prevalencia de la obesidad se mantuvo relativamente estable durante 2 décadas (19601980), sin embargo, aumentó significativamente entre 1976-80 y 1988-94 (aumentó de 12,3\% a 20,6\%) y entre 1988-94 y 1999-2000 (aumentó de $20,6 \%$ a $28,4 \%)^{18,19}$.

A pesar de que las cifras de obesidad en Chile se sitúan entre los primeros lugares del mundo y el grupo de MEF es un grupo estratégico de intervención para prevenir la obesidad y ECNT tanto en la mujer como en su descendencia, no existen estadísticas nacionales sobre la evolución de la obesidad en este grupo, existiendo sólo dos encuestas transversales (Encuesta Nacional de Salud [ENS] 2003 y 2009-2010). Por otro lado, existen estadísticas que describen prevalencias de obesidad en un subgrupo de las MEF correspondiente a las embarazadas atendidas en el sistema público de salud ${ }^{20-22}$, aunque lamentablemente no están incorporadas las embarazadas atendidas en el sistema privado. Además, no es posible comparar la prevalencia de obesidad en gestantes en el período 1987-2004 con el período 2005-2013, por el uso de un estándar de evaluación del estado nutricional diferente en cada uno de estos períodos. Dos estudios previos a esta fecha realizados en el Área Norte de Santiago mostraron que aproximadamente un tercio de las embarazadas presentó exceso de peso $^{23,24}$.

Dados estos antecedentes, el objetivo de este trabajo fue rescatar toda la evidencia disponible en Chile sobre obesidad en MEF. Para tal efecto: 1) Se revisaron los trabajos publicados en Chile sobre prevalencias de obesidad en MEF; 2) Se compararon las cifras de obesidad en MEF entre los años 2003 y 2009-2010; 3). Se analizó la tendencia de la prevalencia de obesidad en embarazadas que se atienden en el sistema de salud público de Chile entre los años 1987 y 2013.

\section{Material y Métodos}

Se utilizaron tres aproximaciones para describir la prevalencia de obesidad en MEF: i) Revisión cualitativa de estudios que describieron las preva- lencias de obesidad en MEF chilenas; ii) Estudio comparativo de las prevalencias de obesidad, sobrepeso, exceso de peso, obesidad abdominal y obesidad mórbida en las mujeres provenientes de las ENS, 2003 y 2009-2010 y iii) Evaluación de la tendencia del estado nutricional de las embarazadas atendidas en el sistema público de salud entre los años 1987 y 2013.

\section{i. Revisión cualitativa}

Se realizó una búsqueda en Medline incluyendo los términos "obesity prevalence" y "Chile". Se encontraron 293 trabajos, de los cuales, se incluyeron 10 en esta revisión que cumplieron con los criterios de inclusión de describir prevalencias de obesidad en MEF no embarazadas y ser realizados en Chile. Una búsqueda en Scielo permitió seleccionar 3 trabajos de 100 encontrados. Además se revisaron otras bases de datos (Lilacs, Cochrane, Embase y Cinahl), en las cuales no se encontraron artículos. Para cada estudio se describió la población total y de MEF, el tamaño y tipo de muestreo, lugar y año de la recolección de los datos, las variables y su operacionalización. La calidad de cada artículo seleccionado se evaluó a través del porcentaje de cumplimiento del total de puntos señalados en la declaración de STROBE ${ }^{25}$. El rango de cumplimiento fluctuó entre $62 \%$ y $73 \%$.

\section{ii. Estudio comparativo de prevalencias de obesidad en MEF}

Se solicitaron al Ministerio de Salud de Chile (MINSAL) las bases de datos de las ENS (2003, 2009-2010). Ambas ENS excluyeron a las embarazadas y fueron representativas de la población nacional según edad, y zona rural-urbana ${ }^{26,27}$. Para fines de este análisis, se seleccionaron las variables edad (17-45 años), peso $(\mathrm{kg})$, talla $(\mathrm{m})$, circunferencia de cintura $(\mathrm{cm})$, obesidad abdominal (circunferencia de cintura $\geq 88 \mathrm{~cm})^{28}$, índice de masa corporal $\left(\mathrm{IMC}=(\mathrm{kg}) /\left(\mathrm{m}^{2}\right)\right)$ y categorías de estado nutricional (sobrepeso (IMC 25-29,9), obesidad (IMC $\geq 30$ ), obesidad mórbida (IMC $>40$ ) y exceso de peso (IMC $\geq 25)^{29}$. El análisis estadístico incluyó porcentajes con sus respectivos intervalos de confianza (95\%) para variables categóricas y promedios y error estándar para variables continuas. La diferencia entre las prevalencias de obesidad entre ambas encuestas se evaluó a través de modelos de regresión logística (Odds Ratio e intervalos de confianza a 95\%) y las 
diferencias entre las variables continuas (cambios en peso, IMC y circunferencia de cintura) a través del estadístico $\mathrm{F}$ de Wald obtenido al realizar un modelo lineal general. Tanto las prevalencias de variables antropométricas como los promedios se ajustaron por nivel educacional y edad de las MEF. Para estos análisis se utilizó el programa SPSS utilizando factores de expansión.

\section{iii. Evaluación de la tendencia de las prevalencias de obesidad en la embarazada}

La categorización antropométrica en gestantes atendidas en el sistema público de salud se obtuvo a partir de informes técnicos del departamento de estadísticas e información en salud del MINSAL (DEIS $)^{20-22}$ y del Departamento de Nutrición de la Facultad de Medicina de la Universidad de Chile.

Entre los años 1987 y 2004, el MINSAL utilizó el estándar de peso para la talla $(\mathrm{P} / \mathrm{T})$ según edad gestacional para evaluar la antropometría de las embarazadas en Chile ${ }^{30}$. Esta establece un rango de peso aceptable para cada edad gestacional expresado como porcentaje de peso para la talla de una mujer no embarazada. Se estimó como incremento de peso ideal 20\% del peso aceptable. Entre los años 2005 y hasta la fecha el MINSAL utiliza el estándar de referencia de IMC según la edad gestacional ${ }^{31}$. Para efecto de este estudio, se graficó el estado nutricional de las embarazadas entre los años 1987 y 2013 y se analizó la tasa de aumento anual de sobrepeso, obesidad y exceso de peso en tres períodos: 1987-1995, 1996-2004, 2005-2011.

\section{Resultados}

\section{i. Revisión cualitativa}

En la década 1980-89, Berríos y col..$^{32}$ mostraron en un estudio realizado en población general que la prevalencia de obesidad en mujeres de 15-24 años era $8 \%$ y que se duplicaba y triplicaba en los grupos de edad de 25-34 y 35-44, respectivamente (Tabla 1).

En la década 1990-99 se encontraron 3 estudios que describieron prevalencias de obesidad fluctuantes, que iban desde $2 \%$ en universitarias de Concepción hasta $21 \%$ en población general de Valparaíso ${ }^{33-35}$ (Tabla 1).

En la década 2000-09, además de las dos ENS que se resumirán más adelante, se encontraron 9 estudios. Cinco de estos estudios se realizaron en población general, dos son de cohorte y tres transversales, alcanzando prevalencias de obesidad más altas a las décadas precedentes, desde $18 \%$ a $35 \%{ }^{36-40}$. Cuatro estudios se realizaron en universitarias, con prevalencias de obesidad entre $0 \%$ y $6 \%^{41-44}$ (Tabla 1$)$

\section{ii. Estudio comparativo de la prevalencia de obesidad según Encuestas Nacionales de Salud}

La prevalencia de obesidad en las MEF superó $20 \%$ en las dos mediciones de la ENS (2003 y 2009-2010), con más de la mitad de las mujeres en esta edad con exceso de peso ambos años. Con excepción de la obesidad mórbida, las prevalencias de obesidad, sobrepeso, exceso de peso y obesidad abdominal fueron más altas en la medición del año 2009-2010 que en la del 2003, pero este incremento no fue estadísticamente significativo, incluso después de ajustar los datos por nivel educacional y edad (Tabla 2).

Los promedios de peso, IMC y circunferencia de cintura también fueron más altos el año 2009 que el año 2003, pero sólo el aumento de $2 \mathrm{~cm}$ en el promedio de la circunferencia de cintura alcanzó a ser estadísticamente significativo (Tabla 3).

\section{iii. Tendencia de la evaluación antropométrica de las mujeres embarazadas en Chile}

En la Figura 1 se muestra la tendencia de la evolución antropométrica de las embarazadas en Chile, la cual se estimó desde 1987 al 2004 con estándar $\mathrm{P} / \mathrm{T}^{30}$ y desde el 2005 en adelante con estándar de IMC ${ }^{31}$. Durante el período 19872004 se observó un aumento importante de la obesidad, desde $13 \%$ a $32 \%$ respectivamente, lo que corresponde a $150 \%$ de aumento respecto del valor basal, mientras que el sobrepeso aumentó de $17 \%$ a $23 \%$ en el mismo período. Paralelamente, se observó una disminución de las embarazadas con diagnóstico de bajo peso (desde $26 \%$ a $12 \%$ ) y con estado nutricional normal (desde $44 \%$ a $33 \%$ ) $^{20}$.

Entre los años 2005 y 2013, disminuyeron las embarazadas con estado nutricional normal, de $41 \%$ a $35 \%$. Por el contrario, el sobrepeso y la obesidad aumentaron, de $31 \%$ a $33 \%$ y de $20 \%$ a $26 \%$ respectivamente ${ }^{21-22}$.

La tasa de aumento anual de la prevalencia de obesidad fue mucho mayor entre los años 19871995 (10\%) que en los otros períodos analizados, duplicándose la prevalencia de obesidad al final de este período (Tabla 4). Para los otros dos períodos, 
Tabla 1. Prevalencia de obesidad en mujeres chilenas en edad fértil

\begin{tabular}{|c|c|c|c|c|}
\hline Autor/año/ciudad & $\begin{array}{l}\text { Diseño/Muestreo/Población/ } \\
\text { año de recolección de los } \\
\text { datos }\end{array}$ & Muestra & Variables ${ }^{\&}$ & $\begin{array}{l}\text { Prevalencia (\%) } \\
\text { MEF, (edad) }\end{array}$ \\
\hline $\begin{array}{l}\text { Berríos } X^{32} 1990 \\
\text { Región Metropolitana }\end{array}$ & $\begin{array}{l}\text { Transversal/conglomerado } \\
\text { bi-etápico y estratificado/ } \\
\text { población general/1986-87 }\end{array}$ & $\begin{array}{l}\text { Total }=1.203 \\
\mathrm{MEF}=481 \\
(15-44 \text { años })\end{array}$ & $\mathrm{OB} I \mathrm{IMC} \geq 27$ & $\begin{array}{rr}7,6 \% & (15-24) \\
17,3 \% & (25-34) \\
24,4 \% & (35-44)\end{array}$ \\
\hline $\begin{array}{l}\text { Jadue L } \mathrm{L}^{34} 1999 \\
\text { Valparaíso }\end{array}$ & $\begin{array}{l}\text { Transversal/aleatorio simple } \\
\text { población general/1996-97 }\end{array}$ & $\begin{array}{l}\text { Total }=3.120 \\
\mathrm{MEF}=1.081\end{array}$ & $O B^{*}$ & $\begin{array}{ll}14,4 \% & (25-34) \\
21,4 \% & (35-44)\end{array}$ \\
\hline $\begin{array}{l}\text { Chiang-Salgado } 35 \\
1999 \text { U de } C^{\searrow}\end{array}$ & $\begin{array}{l}\text { Transversal/aleatorio en múltiples } \\
\text { etapas/Universitarios/1998 }\end{array}$ & $\begin{array}{c}\text { Total }=1.301 \\
\mathrm{MEF}=564\end{array}$ & $\mathrm{OB} I \mathrm{IMC}>30$ & $2,2 \% \quad(18-25)$ \\
\hline $\begin{array}{l}\text { Bustos P36 } 2003 \\
\text { Limache }\end{array}$ & $\begin{array}{l}\text { Cohorte/aleatorio/Mujeres } \\
\text { nacidas entre 1974-78/2000-02 }\end{array}$ & $\begin{array}{l}\text { Total }=850 \\
\text { MEF }=465\end{array}$ & $\begin{array}{l}\mathrm{OB}^{*} \\
\mathrm{SP}^{*}\end{array}$ & $\begin{array}{ll}17,5 \% & (22-28) \\
31,6 \% \quad(" & (")\end{array}$ \\
\hline $\begin{array}{l}\text { Blümel J33 } 2003 \\
\text { SSMS** }\end{array}$ & $\begin{array}{l}\text { Cohorte/total de mujeres } \\
\text { atendidas en unidad de medicina } \\
\text { preventiva/1991-92 }\end{array}$ & $\begin{array}{l}\text { Total }=576 \\
\mathrm{MEF}=140\end{array}$ & $\begin{array}{l}\mathrm{OB}^{*} \\
\mathrm{SP}^{*}\end{array}$ & $\begin{array}{rr}8,6 \% & (40-44) \\
39,3 \% & (" \quad ")\end{array}$ \\
\hline $\begin{array}{l}\text { ENS } 2003^{26} \\
\text { Chile }\end{array}$ & $\begin{array}{l}\text { Transversal/muestreo aleatorio } \\
\text { bifásico/Población general/2003 }\end{array}$ & $\begin{array}{c}\text { Total }=3.619 \\
\mathrm{MEF}=827\end{array}$ & $\begin{array}{l}\mathrm{OB}^{*} \\
\mathrm{SP}^{*}\end{array}$ & $\begin{array}{ll}21,0 \% & (17-45) \\
30,8 \% & (17-45)\end{array}$ \\
\hline $\begin{array}{l}\text { Palomo } 1^{38} 2007 \\
\text { Talca }\end{array}$ & $\begin{array}{l}\text { Transversal/polietápico (simple y } \\
\text { sistemático)/Población general/ } \\
2005\end{array}$ & $\begin{array}{c}\text { Total }=1.136 \\
\mathrm{MEF}=317\end{array}$ & $\begin{array}{l}\mathrm{OB}^{*} \\
\mathrm{SP}^{*}\end{array}$ & $\begin{array}{ll}22,1 \% & (18-44) \\
36,6 \% & (18-44)\end{array}$ \\
\hline $\begin{array}{l}\text { Nozue } \mathrm{M}^{37} 2007 \\
\text { San Carlos }\end{array}$ & $\begin{array}{l}\text { Transversal/ND/Población general/ } \\
2004\end{array}$ & $\begin{array}{l}\text { Total }=603 \\
\text { MEF }=183 \\
(20-39 \text { años })\end{array}$ & $\begin{array}{l}\mathrm{OB}^{*} \\
\mathrm{SP}^{*}\end{array}$ & $\begin{array}{ll}35,3 \% & (20-43) \\
41,6 \% & (20-43)\end{array}$ \\
\hline $\begin{array}{l}\text { Mardones H41 } 2009 \\
\text { U. del Bío Bío }\end{array}$ & $\begin{array}{l}\text { Transversal/estratificado/ } \\
\text { Universitarios/ ND }\end{array}$ & $\begin{array}{l}\text { Total }=955 \\
\mathrm{MEF}=372\end{array}$ & $\begin{array}{l}\mathrm{OB}^{*} \\
\mathrm{SP}^{*}\end{array}$ & $\begin{array}{rr}5,9 \% & (17-34) \\
16,9 \% & (17-34)\end{array}$ \\
\hline $\begin{array}{l}\text { ENS 2009-1027 } \\
\text { Chile }\end{array}$ & $\begin{array}{l}\text { Transversal/estratificado multietá- } \\
\text { pico y conglomerados/Población } \\
\text { general/2009-10 }\end{array}$ & $\begin{array}{l}\text { Total }=5.416 \\
M E F=1.507 \\
(15-45 \text { años })\end{array}$ & $\begin{array}{l}\mathrm{OB}^{*} \\
\mathrm{SP}^{*}\end{array}$ & $\begin{array}{ll}23,6 \% & (17-45) \\
32,1 \% & (17-45)\end{array}$ \\
\hline $\begin{array}{l}\text { Aranguiz } \mathrm{H}^{42} 2010 \\
\text { PUCV', U. de C. }\end{array}$ & $\begin{array}{l}\text { Transversal/Estratificado aleatorio/ } \\
\text { Universitarios/ ND }\end{array}$ & $\begin{array}{c}\text { Total }=64^{\circ} \\
245^{\circ} \\
M E F=44^{\circ} \\
115^{\diamond}\end{array}$ & $\begin{array}{l}\mathrm{OB}^{*} \\
\mathrm{SP}^{*}\end{array}$ & $\begin{array}{cr}0 \% & 2,6 \% \\
20 \% & 13,9 \% \\
(\bar{x}=19,9 \pm 2,3)^{\bullet} \\
(\bar{x}=18,9 \pm 0,9)^{\bullet}\end{array}$ \\
\hline $\begin{array}{l}\text { Ratner } \mathrm{R}^{43} 2012 \\
54 \text { U. del país }\end{array}$ & $\begin{array}{l}\text { Transversal/Voluntarios/Universi- } \\
\text { tarios/2010-2011 }\end{array}$ & $\begin{aligned} \text { Total } & =6.823 \mathrm{MEF} \\
& =4.317\end{aligned}$ & $\begin{array}{l}\mathrm{OB}^{*} \\
\mathrm{SP}^{*}\end{array}$ & $\begin{array}{rr}4,1 \% & (19-29) \\
19,8 \% & (19-29)\end{array}$ \\
\hline $\begin{array}{l}\text { Vera-Villarroel }{ }^{44} 2014 \\
\text { USACH }\end{array}$ & $\begin{array}{l}\text { Transversal/Aleatorio simple/ } \\
\text { Universitarios/ND }\end{array}$ & $\begin{array}{l}\text { Total }=3.311 \\
\mathrm{MEF}=1.493\end{array}$ & $\begin{array}{l}\mathrm{OB}^{*} \\
\mathrm{SP}^{*}\end{array}$ & $\begin{array}{rr}1,3 \% & (17-24) \\
10,3 \% & (17-24)\end{array}$ \\
\hline $\begin{array}{l}\text { Mujica-Coopman } \mathrm{M}^{39} \\
2014\end{array}$ & $\begin{array}{l}\text { Transversal/ND/Población general/ } \\
\text { ND }\end{array}$ & $\begin{array}{l}\text { Total }=\text { ND } \\
\text { MEF }=318\end{array}$ & $\begin{array}{l}\mathrm{OB}^{*} \\
\mathrm{SP}^{*}\end{array}$ & $\begin{array}{l}24(\bar{x}=40 \pm 5) \\
47(\bar{x}=40 \pm 5)\end{array}$ \\
\hline $\begin{array}{l}\text { Garmendia M }{ }^{40} \\
2014\end{array}$ & $\begin{array}{l}\text { Cohorte/Universo/Universo de } \\
\text { madres de niños del estudio } \\
\mathrm{GOCS}^{49} / 2007\end{array}$ & $\begin{array}{c}\text { Total }=1195 \\
\text { MEF }=761\end{array}$ & $\begin{array}{l}\mathrm{OB}^{*} \\
\mathrm{SP}^{*}\end{array}$ & $\begin{array}{l}25,9 \%(\bar{x}=32 \pm 7) \\
35,1 \%(\bar{x}=32 \pm 7)\end{array}$ \\
\hline
\end{tabular}

SSMS = Unidad de Medicina Preventiva del Servicio de Salud Metropolitano Sur •PUCV = Pontificia Universidad Católica de Valparaíso, $\cup$ de $C=$ Universidad de Concepción, USACH = Universidad de Santiago de Chile, ND = no descrito, OB = obesidad, $\mathrm{SP}=$ sobrepeso, $\mathrm{EP}=$ exceso de peso e IMC según OMS $=\left(\mathrm{BP}^{*} \mathrm{IMC}<18,5\right.$, normalidad IMC entre 18,5-24,9, SP* IMC entre 25 y $29,9, \mathrm{OB}^{*} \geq 30$ ), Variables $^{\&}=$ puntos de corte. 
Tabla 2. Comparación de las prevalencias ajustadas de sobrepeso, obesidad y exceso de peso en mujeres en edad fértil de 17 a 45 años entre ENS 2003 y ENS 2009-10

\begin{tabular}{|c|c|c|c|c|c|c|c|}
\hline \multirow[t]{2}{*}{ Condiciones de salud } & \multicolumn{2}{|c|}{ ENS2003 } & \multicolumn{2}{|c|}{ ENS2009-10 } & \multirow[t]{2}{*}{ OR } & \multirow[t]{2}{*}{ IC $95 \%$} & \multirow[b]{2}{*}{ OR aj IC 95\% } \\
\hline & $\%$ & IC $95 \%$ & & IC $95 \%$ & & & \\
\hline Obesidad (IMC $\geq 30$ ) & 21,0 & {$[17,3-25,5]$} & 23,6 & {$[20,1-27,4]$} & 1,2 & {$[0,9-1,6]$} & $1,3[0,9-1,6]$ \\
\hline Sobrepeso (IMC $\geq 25 \leq 29,9$ ) & 30,8 & {$[26,3-35,6]$} & 32,1 & {$[28,2-36,4]$} & 1,1 & {$[0,8-1,4]$} & $1,0[0,8-1,4]$ \\
\hline Exceso de peso (IMC $\geq 25$ ) & 51,3 & {$[46,1-56,5]$} & 55,6 & {$[51,2-59,9]$} & 1,2 & {$[0,9-1,6]$} & $1,2[0,9-1,6]$ \\
\hline Obesidad mórbida (IMC $\geq 40$ ) & 2,2 & {$[1,2-4,1]$} & 2,1 & {$[1,3-3,4]$} & 1,0 & {$[0,4-2,2]$} & $0,7[0,3-1,4]$ \\
\hline Obesidad abdominal $(\mathrm{cm} \geq 88)$ & 35,5 & {$[30,8-40,5]$} & 37,9 & {$[33,8-42,2]$} & 1,1 & {$[0,8-1,5]$} & $1,2[0,9-1,6]$ \\
\hline
\end{tabular}

El número de sujetos para calcular las prevalencias fue de 778 el año 2003 y 1.390 el año 2009. OR aj = Odds Ratio ajustados por nivel educacional ( $<8$ años de educación, entre 8-12 años y > 12 años de educación) y edad (17-29 años, 30-39 años y 40-45 años).

Tabla 3. Comparación de los promedios ajustados de peso, IMC y circunferencia de cintura en mujeres en edad fértil de 17 a 45 años entre ENS 2003 y ENS 2009-10

\begin{tabular}{|lcccccccc|}
\hline Condiciones de salud & $\begin{array}{c}\text { ENS2003 } \\
\overline{\mathbf{x}} \pm \mathbf{E E}\end{array}$ & $\begin{array}{c}\text { ENS2009-10 } \\
\overline{\mathbf{x}} \pm \mathbf{E E}\end{array}$ & $\mathbf{F}$ & $\mathbf{p}$ & $\begin{array}{c}\text { ENS2003 } \\
\overline{\mathbf{x}} \mathbf{a j} \pm \mathbf{E E}\end{array}$ & $\begin{array}{c}\text { ENS2009-10 } \\
\overline{\mathbf{x}} \text { aj } \pm \text { EE }\end{array}$ & F & p \\
\hline Peso $(\mathrm{kg})$ & $65,0 \pm 0,6$ & $66,6 \pm 0,7$ & 3,3 & 0,071 & $65,8 \pm 0,6$ & $67,5 \pm 0,7$ & 3,8 & 0,050 \\
$\mathrm{IMC}\left(\mathrm{kg} / \mathrm{m}^{2}\right)$ & $26,3 \pm 0,3$ & $26,6 \pm 0,2$ & 0,6 & 0,456 & $26,7 \pm 0,3$ & $27,2 \pm 0,3$ & 2,4 & 0,124 \\
Circunferencia cintura $(\mathrm{cm})$ & $83,7 \pm 0,6$ & $85,0 \pm 0,7$ & 2,3 & 0,134 & $84,7 \pm 0,6$ & $86,4 \pm 0,7$ & 4,3 & 0,039 \\
\hline
\end{tabular}

Los promedios fueron ajustados ( $\bar{x}$ aj) por nivel educacional ( $<8$ años de educación, entre 8-12 años y $>12$ años de educación) edad (17-29 años, de 30-39 años y de 40-45 años).

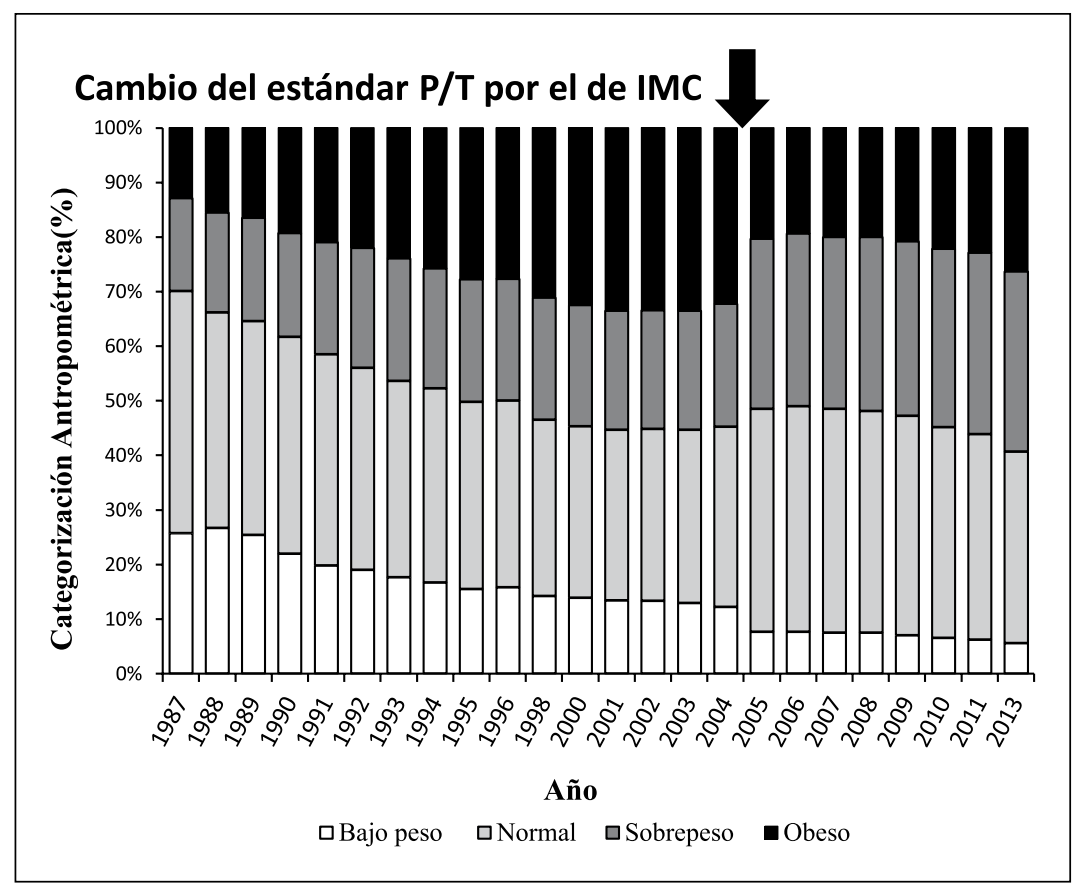

Figura 1. Tendencia y efecto del cambio de estándar en la evaluación antropométrica de las embarazadas atendidas en el sistema público de salud en el período 1987-2013. Medición de acuerdo a estándar peso/ talla para la edad gestacional (19872004) e IMC para la edad gestacional (2005-2013). Los datos del período 1987-1996 se obtuvieron del Departamento de Nutrición de la Facultad de Medicina de la Universidad de Chile y los del año 2011 y 2013 se obtuvieron del Departamento de Estadísticas e Información en Salud (MINSAL). El cambio de estándar coincide con el desarrollo de la estrategia de intervención nutricional a través del ciclo vital impulsada por MINSAL en el año 2005. 
Tabla 4. Prevalencias (\%) y tasas de aumento anual de sobrepeso, obesidad y exceso de peso por período (1987-1995; 1996-2004; 2005-2011) en gestantes atendidas en el sistema público de salud chileno

\begin{tabular}{|c|c|c|c|c|c|c|}
\hline \multirow[t]{2}{*}{ Período } & \multicolumn{2}{|c|}{ Sobrepeso } & \multicolumn{2}{|c|}{ Obesidad } & \multicolumn{2}{|c|}{ Exceso de Peso } \\
\hline & $\begin{array}{c}\text { Prevalencia } \\
\text { (\%) }\end{array}$ & $\begin{array}{c}\text { Tasa de } \\
\text { aumento } \\
\text { anual }(\%)\end{array}$ & $\begin{array}{c}\text { Prevalencia } \\
\text { (\%) }\end{array}$ & $\begin{array}{c}\text { Tasa de } \\
\text { aumento } \\
\text { anual (\%) }\end{array}$ & $\begin{array}{c}\text { Prevalencia } \\
\text { (\%) }\end{array}$ & $\begin{array}{c}\text { Tasa de } \\
\text { aumento } \\
\text { anual (\%) }\end{array}$ \\
\hline 1987 & 17,0 & \multirow{2}{*}{3,5} & 12,9 & \multirow[t]{2}{*}{10,1} & 29,9 & \multirow{2}{*}{6,7} \\
\hline 1995 & 22,4 & & 27,8 & & 50,2 & \\
\hline 1996 & 22,3 & \multirow{2}{*}{0,1} & 27,7 & \multirow{2}{*}{1,9} & 50,0 & \multirow{2}{*}{1,1} \\
\hline 2004 & 22,5 & & 32,2 & & 54,7 & \\
\hline 2005 & 31,2 & \multirow{2}{*}{1,0} & 20,3 & \multirow{2}{*}{2,0} & 51,5 & \multirow{2}{*}{1,5} \\
\hline 2011 & 33,2 & & 22,9 & & 56,1 & \\
\hline
\end{tabular}

En período 1987-2004 se utilizó estándar $\mathrm{P} / \mathrm{T}^{30}$ y en el período 2005-2011 se utilizó el estándar de IMC ${ }^{31}$ para calcular las prevalencias de categorización antropométrica.

1996-2004 y 2005-2011, la tasa de aumento fue de 2\% anual. El período 1987-1995 también fue el de mayor incremento anual tanto para el sobrepeso como para el exceso de peso en relación a los otros dos períodos analizados (Tabla 4).

\section{Discusión}

En este estudio se analizaron para Chile las prevalencias de obesidad y su tendencia en MEF y en el grupo específico de embarazadas atendidas en el sistema público de salud. Para este efecto se revisó toda la evidencia publicada y se analizaron a partir de datos nacionales los cambios en la tendencia de las prevalencias. En relación a los estudios publicados, fue difícil la comparación entre los mismos debido a la heterogeneidad de poblaciones de estudio, diseños, tamaños muestrales, tipos de muestreo y los puntos de corte utilizados para el diagnóstico de obesidad, como en el caso de Berríos que utilizó como punto de corte para obesidad un IMC $\geq 27^{32}$. Pocos de los estudios encontrados se focalizaron específicamente en este grupo etáreo y muestran prevalencias variables, las prevalencias más altas se encontraron en San Carlos en la década 2000-09 y en general en los estudios de base poblacional ${ }^{36-40}$ más que en la población universitaria ${ }^{41-44}$, probablemente asociado a una mayor edad y a un menor nivel educacional.

La ENS 2003 mostró que una de cada 5 mujeres en edad fértil era obesa, mientras que para para el año 2009-2010 fue casi una de cada cuatro. Estas cifras son similares a las cifras publicadas por Mujica y Garmendia en Chile en el año 2014. También son comparables a las publicadas en la región de las Américas y el Caribe (superior a 20\%) en el año $2008^{17}$. Al comparar las prevalencia de obesidad en Chile entre las MEF de 20 a 39 años según ENS con las descritas según NHANES en Estados Unidos de Norteamérica, las prevalencias en Chile fueron menores con 18,5\% (2003) y $24,3 \%$ (2009) vs $28,9 \%$ (NHANES 2003-2004) y $34 \%$ (NHANES 2007-2008) ) $^{18,19}$.

La prevalencia de obesidad en MEF en la ENS 2009-2010 aumentó en 12\% en relación al año 2003 , pero este incremento no fue estadísticamente significativo. Existen varias explicaciones para este hallazgo. En primer lugar, no se descarta que el bajo tamaño muestral en este grupo etáreo sea la explicación para la falta de significancia estadística. En segundo lugar, es posible que el intervalo de tiempo entre ambas mediciones haya sido insuficiente para mostrar cambios en parámetros que dependen de cambios de hábitos o de estilos de vida de la población. Sin embargo, el estudio de cohorte de Garmendia et al mostró en 761 mujeres en edad fértil de nivel socioeconómico medio y bajo incrementos significativos en el peso y en el IMC en sólo tres años de seguimiento. Por otro lado, a pesar de que las ENS son representativas de la realidad de Chile, debido a su carácter transver- 
sal no son totalmente comparables. Finalmente, la intervención nutricional a través del ciclo vital implementada por el MINSAL el año 2005 pudo haber contribuido a evitar un aumento mayor de la obesidad. Lamentablemente no se han encontrado estudios que muestren evaluaciones de impacto de esta estrategia.

Las prevalencias nacionales de obesidad de las embarazadas atendidas en el sistema público de salud fueron $33,5 \%$ el año $2003^{20}$ y $20,8 \%$ el $2009^{22}$. La primera es muy superior a la prevalencia de obesidad encontrada en MEF según ENS 2003 (21\%), en cambio, la prevalencia del año 2009 es más cercana al valor encontrado en las MEF (ENS 2009-2010) (23,6\%). Probablemente uno de los factores que influyeron, es que el año 2003 el punto de corte para diagnosticar obesidad que utilizó el estándar P/T fue > 120\% o IMC > $26,73^{45}$ al inicio del embarazo, mientras que con el estándar de IMC, el año 2009 este fue $>30,2^{31}$, similar al utilizado para evaluar a las MEF. Al tener el estándar peso talla un punto de corte más bajo aumenta la prevalencia de embarazadas obesas que según el estándar de IMC serían clasificadas como sobrepeso. Por otro lado, el punto de corte para definir bajo peso al inicio del embarazo en la curva $\mathrm{P} / \mathrm{T}$ es $<95 \%$ o IMC $<21,15^{45}$, siendo el estándar de $\mathrm{IMC}<20,3^{31}$. El estándar $\mathrm{P} / \mathrm{T}$ se caracteriza por su sensibilidad para detectar embarazadas con diagnóstico de obesidad y bajo peso, mientras el estándar de IMC se caracteriza por su especificidad en estos diagnósticos. Lamentablemente el estado nutricional de la embarazada se determinó para los dos períodos analizados en el nivel primario de atención, por lo que no es posible recalcular las prevalencias de obesidad del período 1987-2004 con estándar utilizado en período 2005-2013 y viceversa, lo que hubiera permitido un adecuado análisis de la tendencia. No es posible asegurar que la disminución de la prevalencia de obesidad en gestantes entre los años 2004 y 2005 se deba exclusivamente al cambio de estándar porque ese año se implementó en todo el país la estrategia nutricional a través del ciclo vital para disminuir la obesidad.

Como conclusión, este estudio mostró prevalencias altas y crecientes de obesidad en las MEF y también en el subgrupo específico de embarazadas atendidas en el sistema público de salud. Las MEF constituyen un grupo estratégico de intervención para quebrar la actual tendencia de aumento de la obesidad, porque de ellas no sólo depende su bienestar actual y futuro sino también el de sus hijos. Monitorear las prevalencias de obesidad y de los entornos alimentarios y de actividad física asociados permitirá evaluar su tendencia y las estrategias de prevención y control.

Agradecimientos: Al Departamento de Epidemiología del Ministerio de Salud de Chile por facilitar las bases de datos de las ENS, al Departamento de Estadísticas e Información en Salud (DEIS-MINSAL) y a Iván Palomo (PhD) por facilitar los datos de su estudio en Talca.

\section{Referencias}

1. Dennedy M, Ávalos G, O’Reilly M, O’Sullivan E, Gaffney G, Dunne F. ATLANTIC-DIP: Raised Maternal Body Mass Index (BMI) Adversely Affects Maternal and Fetal Outcomes in Glucose-Tolerant Women according to International Association of Diabetes and Pregnancy Study Groups (IADPSG) Criteria. J Clin Endocrinol Metab 2012; 97 (4): E608-12.

2. Leung TY, Leung TN, Sahota D, Chan O, Chan L, Fung T, et al. Trends in maternal obesity and associated risks of adverse pregnancy outcomes in a population of Chinese women. BJOG 2008; 115 (12): 1529-37.

3. Powe C, Ecker J, Rana S, Wang A, Ankers E, Ye J, et al. Preeclampsia and the risk of large-for-gestational-age infants. Am J Obstet Gynecol 2011; 204 (5): 425.e1-6.

4. Briese V, Voigt M, Wisser J, Borchardt U, Straube S. Risks of pregnancy and birth in obese primiparous women: an analysis of German perinatal statistics. Arch Gynecol Obstet 2011; 283: 249-53.

5. Lombardi D, Barton J, O’Brien J, Istwan N, Sibai B. Does an obese prepregnancy body mass index influence outcome in pregnancies complicated by mild gestational hypertension remote from term? Am J Obstet Gynecol 2005; 192 (5): 1472-4.

6. Persson M Pasupathy D, Hanson U, Westgren M, Norman M. Pre-pregnancy body mass index and the risk of adverse outcome in type 1 diabetic pregnancies: a population-based cohort study. BMJ Open 2012; 2 (1): e000601.

7. Kyvernitakis I, Köhler C, Schmidt S, Misselwitz B, Großmann J, Hadji P, Kalder M. Impact of maternal body mass index on the cesarean delivery rate in Germany from 1990 to 2012. J Perinat Med 2014. pii: /j/jpme. ahead-of-print/jpm-2014-0126/jpm-2014-0126.xml. doi: 10.1515/jpm-2014-0126. [Epub ahead of print].

8. Nohr EA, Timpson NJ, Andersen CS, Davey Smith 
G, Olsen J, Sørensen TI. Severe Obesity in Young Women and Reproductive Health: The Danish National Birth Cohort. PLoS One 2009; 24: 4 (12): e8444.

9. Hajer G, Van Haeften T, Visseren F. Adipose tissue dysfunction in obesity, diabetes, and vascular diseases. Eur Heart J 2008; 29 (24): 2959-71.

10. Bessonova L, Marshall SF, Ziogas A, Largent J, Bernstein L, Henderson KD, Ma H, West DW, Anton-Culver $\mathrm{H}$. The association of body mass index with mortality in the California Teachers Study. Int J Cancer 2011; 129 (10): 2492-501.

11. Simpson ER, Brown KA. Minireview: Obesity and breast cancer: a tale of inflammation and dysregulated metabolism. Mol Endocrinol 2013. [Epub ahead of print].

12. Bao C, Zhou Y, Jiang L, Sun C, Wang F, Xia W, et al. Reasons for the increasing incidence of macrosomia in harbin, China. BJOG 2011; 118 (1): 93-8.

13. Swank ML, Caughey AB, Farinelli CK, Main EK, Melsop $\mathrm{KA}$, Gilbert WM, Chung JH. The impact of change in pregnancy body mass index on macrosomia. Obesity 2014. doi: 10.1002/oby.20790. [Epub ahead of print].

14. Wang T, Zhang J, Lu X, Xi W, Li Z. Maternal early pregnancy body mass index and risk of preterm birth. Arch Gynecol Obstet 2011; 284 (4): 813-9.

15. O’Reilly JR, Reynolds RM. The risk of maternal obesity to the long-term health of the offspring. Clin Endocrinol (Oxf) 2013; 78 (1): 9-16.

16. Loaiza S, Atalah E. Birth weight and obesity risk at first grade of high school in a non-concurrent cohort of Chilean children. Public Health Nutr 2013; 16 (2): 228-32.

17. Black R, Victora C, Walker S, Bhutta Z, Christian $\mathrm{P}$, de Onis M, et al. The Maternal and Child Nutrition Study Group. Maternal and child undernutrition and overweight in low-income and middleincomecountries. Lancet 2013 doi:pii: S0140-6736 (13) 60937-X. 10.1016/S0140-6736(13)60937-X. [Epub ahead of print].

18. Flegal K, Carroll M, Ogden C, Johnson C. Prevalence and Trends in Obesity Among US Adults, 1999-2000 JAMA 2002; 288: 1723-27.

19. Flegal K, Carroll M, Ogden C, Curtin L. Prevalence and Trends in Obesity Among US Adults, 1999-2008 JAMA 2010; 303 (3): 235-41.

20. Minsal. Evolución de la situación nutricional en Chile 1994-2006. http://www.redsalud.gov.cl/archivos/alimentosynutricion/informe_nut_94_2006.pdf. Consultado el 10 de febrero de 2012.

21. Minsal. Informe de la situación nutricional en el país 2006-2008. http://www.red salud.gov.cl/portal/url/item /6b4d0a9b42e14e04001011e01277c.pdf. Consultado el 10 de febrero de 2012.
22. Minsal. Estado nutricional 2009-2010. http://deis.minsal. cl/ev/en. Consultado el 10 de febrero de 2012.

23. Atalah E, Rosales E, Barja M, Rutman M, Troncoso M, Lagos I. Nutrición materna y crecimiento fetal. Alternativas para Chile. Rev Med Chile 1980; 108: 351-7.

24. Raby E, Atalah E, Cumsille F. Relación entre el peso del recién nacido y variables nutricionales y biomédicas maternas. Rev Chil Nutr 198311 (3): 23-5.

25. Vandenbroucke JP, Von Elm E, Altman DG, Gøtzsche PC, Mulrow CD, et al. Iniciativa STROBE. Strengthening the reporting of observational studies in epidemiology (STROBE): explanation and elaboration]. Gac Sanit 2009; 23 (2): 158.

26. Encuesta Nacional de Salud 2003, Departamento de Epidemiología, Ministerio de Salud de Chile. http:// epi.minsal.cl/epi/html/invest/ens/informefinalens.pdf. Consultado el 10 de febrero de 2012.

27. Encuesta Nacional de Salud 2009-2010, Departamento de Epidemiología, Ministerio de Salud de Chile. http:// www.minsal.gob.cl/portal/docs/page/minsalcl/g_home/ submenu_portada20111/ens2010.pdf. Consultado el 10 de febrero de 2012.

28. Han T, Van Leer E, Seidell J, Lean M. Waist circumference action levels in the identification of cardiovascular risk factors: prevalence study in a random sample. BMJ 1995; 311 (7017): 1401-5.

29. World Health Organization. HTTP:/WWW.euro.who. int/em/what-we-do/health-topics/disease-prevention/ nutrition/a-healthy-lifestyle/body-mass-index-bmi. Consultado el 12 de febrero de 2012.

30. Mardones F, Rosso P. Design of a weight gain chart for pregnant women (Diseño de una curva patrón de incrementos ponderales para la embarazada). Rev Med Chile 1997; 125: 1437-48.

31. Atalah E, Castillo C, Castro R, Aldea A. Propuesta de un nuevo estándar de evaluación nutricional en embarazadas. Rev Med Chile 1997; 125: 1429-36.

32. Berríos X, Jadue L, Zenteno J, Ross M, Rodríguez H. Prevalencia de factores de riesgo de enfermedades crónicas. Estudio en población general de la Región Metropolitana, 1986-1987. Rev Med Chile 1990; 118: 597-604 y 1041-2.

33. Blümel J, Castelo-Branco C, Roncagliolo M, Binfa L, Sarrá S. Factores de riesgo cardiovascular en una cohorte de mujeres de edad media. Rev Med Chile 2003; 131: 381-9.

34. Jadue L, Vega J, Escobar M, Delgado I, Garrido C, Lastra P, Espejo F, Peruga U. [Risk factors for non communicable diseases: methods and global results of the CARMEN program basal survey]. Rev Med Chile 1999; 127 (8): 1004-13. 
35. Chiang-Salgado M, Casanueva-Escobar V, Cid-Cea X, González-Rubilar U, Olate-Mellado P, Nickel-Paredes F, et al. Factores de riesgo cardiovascular en estudiantes universitarios chilenos. Salud Pública Mex 1999; 41: 444-51.

36. Bustos P, Amigo H, Arteaga A, Acosta A, Rona R. Factores de riesgo de enfermedad cardiovascular en adultos jóvenes. Rev Med Chile 2003; 131: 973-80.

37. Nozue M, Miyoshil M, Okumural J, Sánchez H, Andreu J, Kuroiwa C. Prevalence and determinants of obesity and dietary habits among adults in rural area, Chile Bio Science Trends 2007; 1 (3): 140-8.

38. Palomo I, Icaza G, Mujica V, Núñez L, Leiva E, Vásquez $\mathrm{M}$, et al. Prevalencia de factores de riesgo cardiovascular clásicos en población adulta de Talca, Chile, 2005. Rev Med Chile 2007; 135: 904-12.

39. Mujica-Coopman MF, Brito A, López de Romaña D, Pizarro F, Olivares M. Body mass index, iron absorption and iron status in childbearing age women. J Trace Elem Med Biol 2014. pii: S0946-672X(14)00054-6. doi: 10.1016/j.jtemb. 2014.03.008. [Epub ahead of print].

40. Garmendia ML, Alonso FT, Kain J, Uauy R, Corvalán C. Alarming weight gain in women of a post-transitional country. Public Health Nutr 2014; 17 (3): 667-73. doi:
10.1017/S1368980013000098. Epub 2013 Feb 6.

41. Mardones H, Olivares S, Araneda J, Gómez N. Etapas del cambio relacionadas con el consumo de frutas y verduras, actividad física y control del peso en estudiantes universitarios chilenos. Arch Latinoam Nutr 2009; 59 (3): 304-9.

42. Aránguiz H, García V, Rojas S, Salas C, Martínez R, Mac Millan N. Estudio descriptivo, comparativo y correlacional del estado nutricional y condición cardiorrespiratoria en estudiantes universitarios de Chile. Rev Chil Nutr 201037 (1): 70-8.

43. Ratner R, Hernández P, Martel J, Atalah E. Calidad de la alimentación y estado nutricional en estudiantes universitarios de 11 regiones de Chile Rev Med Chile 2012; 140 (12): 1571-9. doi: 10.4067/S003498872012001200008.

44. Vera-Villarroel P, Piqueras JA, Kuhne W, Cuijpers P, van Straten A. Differences between men and women in self-reported body mass index and its relation to drug use. Subst Abuse Treat Prev Policy 2014; 9:1. doi: 10.1186/1747-597X-9-1.

45. Mardones F, Rosso P. A weight gain chart for pregnant women designed in Chile. Matern Child Nutr 2005; 1 (2): 77-90 